\title{
AUTOMATIC APPLE TREE BLOSSOM ESTIMATION FROM UAV RGB IMAGERY
}

\author{
A. Tubau Comas, J. Valente, L. Kooistra
}

Laboratory of Geo-Information Science and Remote Sensing, Wageningen University \& Research, Droevendaalsesteeg 3, 6708 PB Wageningen, The Netherlands - (joao.valente,aina.tubaucomas, lammert.kooistra)@wur.nl.

KEY WORDS: Thinning, Apple orchard, flowering intensity, UAV, image segmentation

\begin{abstract}
:
Apple trees often produce high amount of fruits, which results in small, low quality fruits. Thinning in apple orchards is used to improve the quality of the apples by reducing the number of flowers or fruits the tree is producing. The current method used to estimate how much thinning is necessary is to measure flowering intensity, currently done by human visual inspection of trees in the orchard. The use of images of apple trees from ground-level to measure flowering intensity and its spatial variation through orchards has been researched with promising results. This research explores the potential of UAV RGB high-resolution imagery to measure flowering intensity. Image segmentation techniques have been used to segment the white pixels, which correspond to the apple flowers, of the orthophoto and the single photos. Single trees have been cropped from the single photos and from the orthophoto, and correlation has been measured between percentage of white pixels per tree and flowering intensity and between percentage of white pixels per tree and flower clusters. The resulting correlation is low, with a maximum of 0.54 for the correlation between white pixels per tree and flower clusters when using the ortophoto. Those results show the complexity of working with drone images, but there are still alternative approaches that have to investigated before discarding the use of UAV RGB imagery for estimation of flowering intensity.
\end{abstract}

\section{INTRODUCTION}

Thinning ensures the production of less but bigger fruits, with better qualities (Link, 2000). Furthermore, in biennial bearing varieties, apple varieties which produce a large amount of fruits one year and little the next year, a continuous yield can be obtained by "trespassing" the thinned fruits to the next year's yield (Pflanz et al., 2016; Greene and Costa, 2013). When the apple tree produces a large number of flowers, chemical or machine thinning is applied to reduce the number of flowers. If many flowers are pollinated, chemical or mechanical thinning can be applied again on young fruitlets before June drop (natural abscission of part of the apples that started to grow). After the June drop, if there are still more fruits than desired, the fruits can be hand thinned because there is no chemical or mechanical thinning options for this stage.

With accurate spatial information about flowering intensity in the orchard, thinning can be optimized for the spatial variation of the orchard by applying different amount of chemical thinning according to the tree's flowering intensity, because the total number of flowers indicates the maximum number of fruits that can be produced each year. This would reduce the input of chemicals in the crop and would reduce or avoid having trees with unequal bearing of fruits, and consequently also hand thinning. Furthermore, Aggelopoulou et al. (2010) points out that spatial knowledge of flower density in an apple orchard can provide information that can help the farmer take better management decisions regarding not only thinning, but also fertilizing and harvesting. With knowledge regarding spatial variability the farmer can use the information to decide to use fertilizer where it is more needed, and use less fertilizer where it is less needed, in trees carrying a lower amount of fruits.

Flowering intensity has traditionally been measured by experts looking at each tree individually, but more modern techniques can be found in computer vision and machine learning approaches. Liakos et al. (2017), Aggelopoulou et al. (2011) and Hočevar et al. (2014) used thresholding techniques to detect pixels that belong to flowers. Diaz et al. (2018) combined Neural
Networks (NN) and a Support Vector Machine (SVM), which is a supervised classifier, to identify individual apple flowers. NN have also been used with promising results to estimate yield from mature apples (Bargoti and Underwood, 2017) and fruitlets (Cheng et al., 2017). And multispectral images including the Near InfraRed (NIR) band were used by Liakos et al. (2017) and Xiao et al. (2014) to derive the Normalised Difference Vegetative Index (NDVI), which was used for the detection of apple flowers.

Few researches have focused on the optimization of thinning in apple orchards, and those that did it mostly used views from the ground level. This research will explore the use of RGB-UAV imagery to predict flowering intensity with the objective to develop a computer vision algorithm that computes the orchards' flowering intensity based on an orthophoto. Furthermore, it will also check the possibility to predict flowering intensity in apple orchards based on drone photos.

\section{STUDY AREA}

This research was conducted with data from an apple orchard located in Randwijk, The Nederlands (Figure 1). The orchard, of 0.47 ha, contains the apple variety Elstar with M9 rootstock with tree spacing of $1 \mathrm{~m}$ and a pollination tree every $10 \mathrm{~m}$. In case there are many flowers, ATS (ammonium thiosulphate) is used for chemical thinning, in case of high pollination and a high number of fruits, Brevis ${ }^{\circledR}$ is used for chemical thinning of fruits with a size between 8 and $16 \mathrm{~mm}$. After June drop, trees are hand thinned until each tree has 100 fruits. 


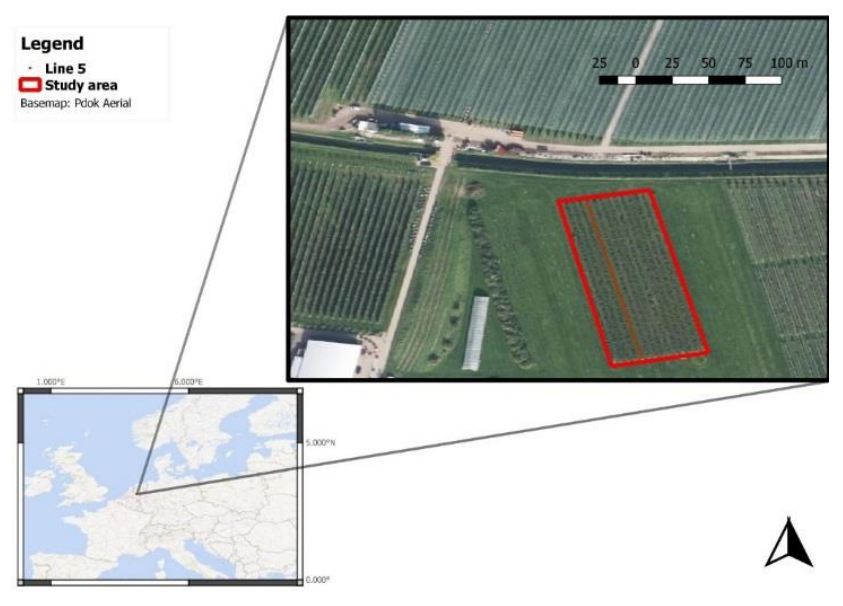

Figure 1. Map of study area

\section{METHODOLOGY}

A drone flight was conducted when the trees were blooming on May $24^{\text {th }}, 2018$ with a rotor UAV (DJI Phantom 3 PRO, Shenzhen, China) (Table 1) equipped with a digital color camera (FC300X, Shenzhen, China) (Table 2), which was flown at $15 \mathrm{~m}$ height to obtain UAV imagery. 356 images were obtained, with an overlap of $85 \%$ and were processed in Agisoft Photoscan, were they were mosaicked into an orthophoto with $4.13 \mathrm{~mm}$ resolution.

\begin{tabular}{|ll|}
\hline Parameters & Specifications \\
\hline Aircraft weight & $1280 \mathrm{~g}$ \\
\hline Number of rotors & 4 \\
\hline Max. Payload & $400 \mathrm{~g}$ \\
\hline Max. flying time & $23 \mathrm{~min}$ \\
\hline Battery & $6000 \mathrm{mAh} \mathrm{LiPo} 2 \mathrm{~S}$ \\
\hline Flying altitude & $10 \mathrm{~m}$ \\
\hline Flying velocity & $2 \mathrm{~ms}^{-1}$ \\
\hline Mission time & $10 \mathrm{~min}^{\mathrm{m}}$ \\
\hline
\end{tabular}

Table 1. UAV parameter specifications

\begin{tabular}{|ll|}
\hline Parameters & Specifications \\
\hline Model & FC300X \\
\hline Sensor resolution & $4000 \times 3000$ \\
\hline Sensor type & CMOS \\
\hline Sensor size & $6.16 \times 4.62 \mathrm{~mm}$ \\
\hline F-stop & $\mathrm{f} / 2.8$ \\
\hline Exposure time & $1 / 1000 \mathrm{~s}$ \\
\hline ISO & 100 \\
\hline Focal length & $3.61 \mathrm{~mm}$ \\
\hline
\end{tabular}

Table 2. Camera parameter specifications

On May $25^{\text {th }}$ ground truth was gathered, which consists of a manual counting of the number of flower clusters in 15 trees and the expert's flowering intensity (floridity) value for 62 trees, given in a scale from 1 to 9 , in which 1 indicates low amount of flowers and 9 high amount of flowers.

In order to use floridity as ground truth to predict flower intensity, the correlation between floridity and flower clusters was checked, which resulted in a $\mathrm{R}^{2}$ of 0.87 , indicating they are highly correlated.
The ground truth was taken only from one row, which consisted of 62 trees; That row was cropped from the orthophoto, and divided in 62 equally sized boxes, each box representing one tree. From this data, the information of the last six trees (57-62) was not used because the quality of the orthophoto in that area was low and could not recreate those trees. For the 15 trees containing data regarding the number of flower clusters, besides from the automatically generated boxes, the trees were manually delineated and cropped out from the orthophoto. Furthermore, five photos containing row five from tree number 1 to tree number 40 seen from a side view were selected, and the trees where manually cropped out of the images (Table 3 ).

\begin{tabular}{|l|lc|} 
& \multicolumn{1}{c}{ Name } & Tree range \\
\cline { 2 - 3 } & DJI_0328.JPG & $1-8$ \\
\cline { 2 - 3 } & DJI_0031.JPG & $9-17$ \\
\cline { 2 - 3 } $\begin{array}{c}\text { Table 3. } \\
\text { the photo } \\
\text { of trees } \\
\text { extracted }\end{array}$ & DJI_0024.JPG & $18-24$ \\
\cline { 2 - 3 } & DJI_0014.JPG & $25-32$ \\
\cline { 2 - 3 } & DJI_0256.JPG & $33-40$ \\
\cline { 2 - 3 } & &
\end{tabular}

Name of and range that were from it

The performance of several segmentation algorithms was compared to detect white pixels. Manual thresholding was used with RGB, HSV and LAB colour spaces. Furthermore, two automatic segmentation methods were used: Otsu's automatic threshold segmentation, which uses the histogram of the image, and assuming a bimodal histogram separates two classes minimizing the variance between classes (Otsu, 1979), and k-means segmentations, which is a clustering algorithm, which finds natural groups. $\mathrm{K}$-means partitions the data into $\mathrm{k}$ groups while minimizing the within-cluster variance (the squared distance between the value of each centre and the value of its assigned data point). K-means was conducted for $\mathrm{k}=2$, which clusters white and background from the original RGB image. Finally, the methodology described in Liakos et al. (2017) (InverseL) was also tested, which consist in using formula 1 to obtain $\mathrm{L}$, calculate the inverse value of $\mathrm{L}$ based on a 255 scale and set a threshold to 100 .

$L=0.21 \times R+0.72 \times G+0.07 \times B$

$$
\begin{array}{ll}
\text { Where } & \mathrm{R}=\text { red } \\
\mathrm{G}=\text { green } \\
\mathrm{B}=\text { blue }
\end{array}
$$

Correlation was tested between percentage of white pixels per tree and flowering intensity and between percentage of white pixels and flower clusters.

\section{RESULTS}

Segmentation was visually tested, and all methods segmented white pixels only, except for InverseL, which segmented white and yellow pixels. From the three colour spaces used, the only one requiring only one band to segment white was RGB, which could segment the white pixels with the blue band. As the automatic thresholding approaches used are based on a single band, they were only conducted with the blue band as input. The tested correlations had consistently a higher $\mathrm{R}^{2}$ when using the automatic segmentation approaches (Otsu and k-means), which are also the segmentation algorithms that results in a higher number of pixels classified as white (Figure 2). 


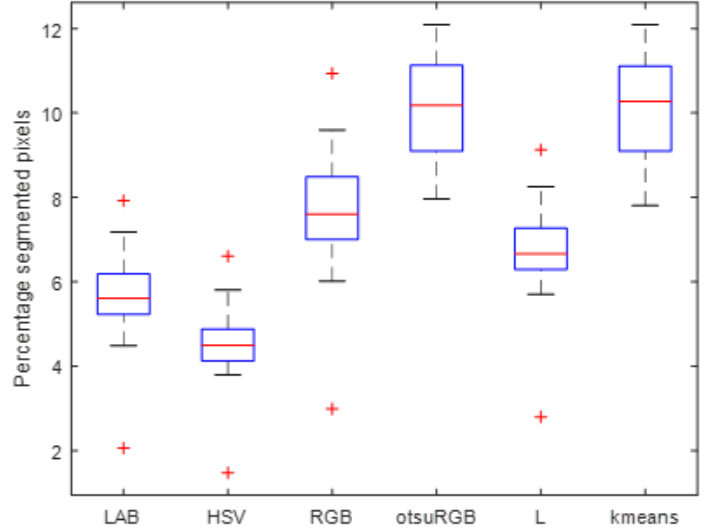

Figure 2. Boxplot showing the percentage of segmented pixels for 20 sub-images extracted from the orthophoto for the different segmentation methods used: manual thresholding for LAB,

HSV and RGB colour spaces, otsu and kmeans for the Blue band and inverse $\mathrm{L}$ threshold method

The $\mathrm{R}^{2}$ obtained for correlation between flowering intensity and percentage of white pixels using Otsu's segmentation was 0.14 when the orthophoto results were used and 0.41 when the results based on the UAV photos were used (Figure 3 ). The $\mathrm{R}^{2}$ obtained for correlation between flower clusters and percentage of white pixels using Otsu's segmentation was 0.54 when the manually selected areas of the orthophoto were used, 0.46 when the automatic orthophoto results were used and 0.53 when the results based on the UAV photos were used (Figure 4).

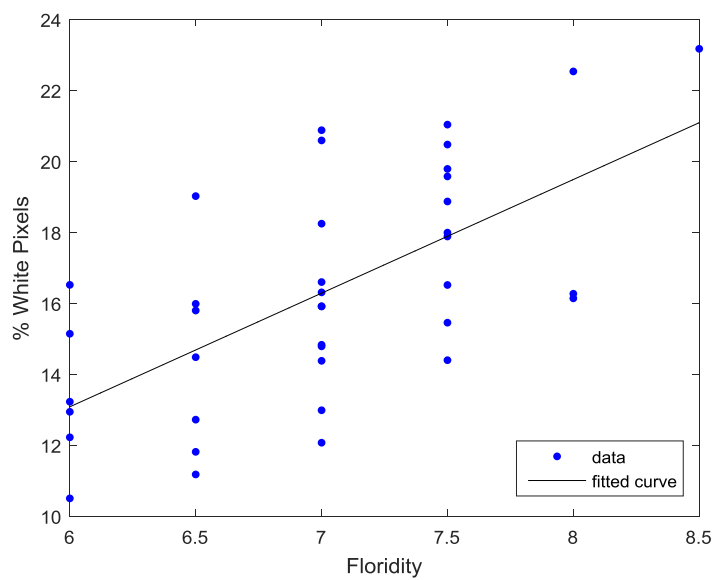

Figure 3. Correlation between floridity in the $\mathrm{x}$ axis and percentage of white pixels (using Otsu's segmentation algorithm in the photos) in the $y$ axis. $R^{2}$ is 0.409 .

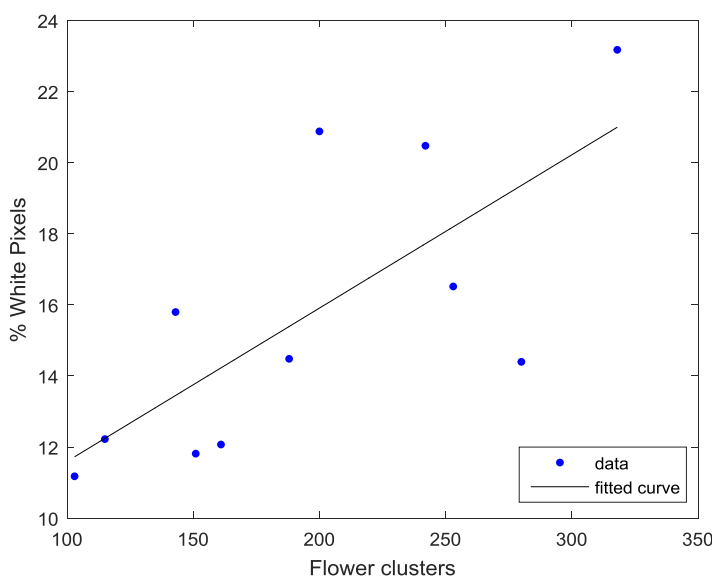

Figure 4. Correlation between number of flower clusters in the tree in $\mathrm{x}$ axis and percentage of white pixels (using Otsu's seg-

mentation algorithm in the photos) in y axis. $\mathrm{R}^{2}$ is 0.532 .

The resolution of the images of the trees cropped from photos differs per picture, nevertheless, this factor does not seem to influence the results. While images of trees from photo DJI_0031.JPG have very similar resolution to those from image DJI_0014.JPG, the first yields very low correlation while the last yields very high correlation between the percentage of white pixels per tree and the floridity value (Table 4).

\begin{tabular}{|cc|c|}
\hline \multicolumn{1}{|c}{ Photo } & Average resolution & $\mathbf{R}^{2}$ Floridity \\
\hline DJI_0328.JPG & $600 \times 320$ & 0.153 \\
\hline DJI_0031.JPG & $314 \times 161$ & 0.037 \\
\hline DJI_0024.JPG & $286 \times 130$ & 0.647 \\
\hline DJI_0014.JPG & $329 \times 154$ & 0.890 \\
\hline DJI_0256.JPG & $492 \times 232$ & 0.131 \\
\hline
\end{tabular}

Table 4. Resolution and $\mathrm{R}^{2}$ for trees in individual pictures

\section{DISCUSSION}

The results of the comparison between segmentation algorithms show that automatic thresholding using Otsu and k-means could provide more accurate segmentation of flowers than manual segmentation. Furthermore, the differences between the different manual thresholding results show that when segmentation is performed manually the number of pixels segmented can change significantly without it implying huge visual differences in the results, making it difficult to set the right threshold. Results also show limitations of InverseL, as it is unsuitable for the dataset at hand, due to the fact that it included yellow pixels in the segmentation of white. This result provides an example of what can happen when the same manual threshold levels set for a specific dataset is used in different data. Therefore, it shows that manual thresholding requires a check and correction of the threshold level every time it is used in a different environment, but also in different lighting conditions, making it less preferable than automatic methods. Even though it has been seen that HSI can be used in variable lighting conditions (Tang et al., 2000), which makes it a preferable choice for images in outdoor agricultural scenes, its relative HSV performed poorly in this dataset because it would include yellow pixels before including all the flower pixels. Instead, the best performing colour space was RGB, specifically, the blue band, which could be used on its own to segment the pixels corresponding to flowers without including yellow pixels. 
There is not abundant literature that aims to predict flowering intensity, which makes our results difficult to compare. For example, Liakos et al. (2017) and Aggelopoulou et al. (2011) used segmentation of flower pixels in images to predict yield but did not provide results that indicate how well their methods detect flowering intensity or number of flowers. Dias et al. (2018) achieved precision rates higher than $90 \%$ for the detection of flowers and Hočevar et al. (2014) got a maximum $\mathrm{R}^{2}$ of 0.59 for the prediction of number of flower clusters.

The low $\mathrm{R}^{2}$ in the results based on the automatic method for the orthophoto could be influenced by:

- Top view of the trees in orthophotos, which is a very different angle from the one that the expert has when providing the ground truth.

- Wind during the UAV flight, which can cause blurry images, because of the small size of the flowers from the apple tree.

- Trees not correctly included in the created boxes, because there are inclined trees and other reasons.

The UAV photos are more similar to the methods used in literature, as the angle is similar (view from the side) and the images are not blurry, as happened in the orthophoto. The $\mathrm{R}^{2}$ for this method reaches 0.53 , which is similar, but still lower than in existing literature. This is an important result because it shows that using UAV images results similar to those obtained by the use of ground photos can be obtained. And these results were obtained with images with lower resolution than those of literature, as each UAV picture do not only show one tree, but many. However, the results for flower clusters are only based on 11 points and must therefore be taken very carefully.

The automatic method using the orthophoto resulted in a low correlation with ground truth, and better results were only obtained with the non-automatic methods, which required of manual cropping of the areas containing trees. A method for automatic single tree detection for apple orchards could be useful to improve the automatic results. Satisfactory results for single tree detection from UAV based on digital terrain model (DTM) have already been obtained in and in citrus orchards (Ok and Ozdarici-Ok, 2018), but dense apple orchards such as the one used in this study present a more challenging scenario for automatic tree detection because trees are so close to each other that it is difficult to differentiate where a tree ends and the next starts. On the other hand, single tree detection has also been applied in complex scenarios such as forests (Mohan et al., 2017; Hirschmugl et al., 2007).

Besides from the orthophoto and the DTM, another derivative from UAV imagery that could be used to predict flowering intensity are structure from motion (SfM) point clouds, which have been used in orchards to study tree structure parameters such as LAI (Mathews and Jensen, 2013), canopy area and tree height (Torres-Sánchez et al., 2018). No relevant literature was found for flower density prediction or similar topics using SfM point clouds, nevertheless, the ability of this method to produce high quality point clouds has been proven multiple times (Leberl et al., 2010; Dandois and Ellis, 2013), making it a good candidate for the task at hand. The main advantage of $3 \mathrm{D}$ point clouds is that they show the tree from all the angles, giving a better representation of it than the $2 \mathrm{D}$ views. In the orchard's case, results could differ if a 2D view is used from one of the sides, if it is taken from the other side and if it is taken from the top. 3D point clouds, on the other hand, include all this information.

\section{CONCLUSIONS AND RECOMENDATIONS}

- Automatic segmentation of white pixels can provide results equal or better than manual threshold segmentation. From the investigated methods, Otsu thresholding with the blue band was found to be the method that detects more white pixels without including yellow pixels in the segmentation. K-means produce very similar results to those obtained using Otsu thresholding algorithm.

- The automatic method using the orthophoto did not provide representative information of the flowering intensity with respect to the ground truth. The same method could be tried in less dense orchards, where distances between trees are higher, or with the use of automatic single tree identification methods based on UAV imagery.

- $\quad$ Alternative methods based on UAV RGB imagery such as $3 \mathrm{D}$ point clouds should be investigated before ruling out the possibility of using UAV RGB imagery to predict flowering intensity.

\section{ACKNOWLEDGEMENTS}

This work was supported by the SPECTORS project (143081) which is funded by the European cooperation program INTERREG Deutschland-Nederland. Moreover, the authors would like to thank Pieter van Dalfsen and Peter Frans de Jong, from Wageningen Plant Research, for providing assistance during the field work at the orchard and for sharing their knowledge that helped us understand more about the apple orchard lifecycle.

\section{REFERENCES}

Aggelopoulou, K.D., Bochtis, D., Fountas, S., Gemtos, T.A., 2011. Yield prediction in apple orchards based on image processing. Precision Agriculture, 12(3), 448-456 https://doi.org/10.1007/s11119-010-9187-0.

Aggelopoulou, K.D., Wulfsohn, D., Fountas, S., Gemtos, T.A., Nanos, G.D., Blackmore, S., 2010. Spatial variation in yield and quality in a small apple orchard. Precision Agriculture, 11, 538556. https://doi.org/10.1007/s11119-009-9146-9.

Bargoti, S. and Underwood, J.P., 2017. Image Segmentation for Fruit Detection and Yield Estimation in Apple Orchards. Journal of Field Robotics, 34(6), 1039-1060.

https://doi.org/10.1002/rob.21699.

Cheng, H., Damerow, L., Sun, Y., Blanke, M., 2017. Early Yield Prediction Using Image Analysis of Apple Fruit and Tree Canopy Features with Neural Networks. Journal of Imaging, 3(6), 1-13. https://doi.org/10.3390/jimaging3010006.

Dandois, J. and Ellis, E., 2013. High spatial resolution three-dimensional mapping of vegetation spectral dynamics using computer vision. Remote Sensing of Environment, 136, 259-276. https://doi.org/10.1016/j.rse.2013.04.005. 
Dias, P.A., Tabb, A., Medeiros, H., 2018. Apple flower detection using deep convolutional networks. Computers in Industry, 99 , 17-28. https://doi.org/10.1016/j.compind.2018.03.010.

Greene, D. and Costa, G., 2013. Fruit Thinning in Pome- and Stone-Fruit: State of the Art. Acta Horticulturae, 998(201307), 93-102. https://doi.org/10.17660/ActaHortic.2013.998.10.

Hirschmugl, M., Ofner, M., Raggam, J., Schardt, M., 2007. Single tree detection in very high resolution remote sensing data. Remote Sensing of Environment, 110, 533-544. https://doi.org/10.1016/j.rse.2007.02.029.

Hočevar, M., Širok, B., Godeša, T., Stopar, M., 2014. Flowering estimation in apple orchards by image analysis. Precision Agriculture, 15(4), 466-478. https://doi.org/10.1007/s11119-0139341-6.

Leberl, F., Irschara, A., Pock, T., Meixner, P., Gruber, M., Scholz, S., Wiechert, A., 2010. Point clouds: Lidar versus 3D vision. Photogrammetric Engineering and Remote Sensing, 76, 1123-1134.

Liakos, V., Tagarakis, A., Aggelopoulou, K., Fountas, S., Nanos, G.D., Gemtos, T., 2017. In-season prediction of yield variability in an apple orchard. European Journal of Horticultural Science, 82(5), 251-259.

https://doi.org/10.17660/eJHS.2017/82.5.5.

Link, H., 2000. Significance of flower and fruit thinning on fruit quality. Plant Growth Regulation, 31, 17-26. https://doi.org/10.1023/A:1006334110068.

Mathews, A. and Jensen, J., 2013. Visualizing and quantifying vineyard canopy lai using an unmanned aerial vehicle (uav) collected high density structure from motion point cloud. Remote Sensing, 5(5), 2164-2183. https://doi.org/10.3390/rs5052164.

Mohan, M., Silva, C.A., Klauberg, C., Jat, P., Catts, G., Hudak, A.T., Dia, M., 2017. Individual Tree Detection from Unmanned Aerial Vehicle (UAV) Derived Canopy Height Model in an Open Canopy Mixed Conifer Forest. Forests, 8(340), 1-17. https://doi.org/10.3390/f8090340.

Ok, A. and Ozdarici-Ok, A., 2018. 2-D delineation of individual citrus trees from UAV-based dense photogrammetric surface models. International Journal of Digital Earth, 11(6), 583-608. https://doi.org/10.1080/17538947.2017.1337820.

Otsu, N., 1979. A Threshold Selection Method from Gray-Level Histograms. IEEE Transactions on Systems, Man, and Cybernetics, 9(1), 62-66. https://doi.org/10.1109/TSMC.1979.4310076.

Pflanz, M., Gebbers, R., Zude, M., 2016. Influence of treeadapted flower thinning on apple yield and fruit quality considering cultivars with different predisposition in fructification. Acta Horticulturae, 1130(201612), 605-612.

https://doi.org/10.17660/ActaHortic.2016.1130.90.

Tang, L., Tian, L., Steward, B.L., 2000. Color image segmentation with genetic algorithm for in-field weed sensing. Transactions- American Society of Agricultural Engineers, 43(4), 10191027.
Torres-Sánchez, J., De Castro, A., Peña, J., Jiménez-Brenes, F., Arquero, O., Lovera, M., López-Granados, F., 2018. Mapping the 3D structure of almond trees using UAV acquired photogrammetric point clouds and object-based image analysis. Biosystems Engineering, 176, 172-184. https://doi.org/10.1016/j.biosystemseng.2018.10.018.

Xiao, C., Zheng, L., Sun, H., Zhang, Y., Li, M., 2014. Estimation of the Apple Flowers Based on Aerial Multispectral Image. American Society of Agricultural and Biological Engineers Annual International Meeting 2014, ASABE 2014, 6, 4426-4433. 